\title{
Normalización de datos geoespaciales de fuentes y manantiales inventariados en el Nomenclátor Geográfico de Andalucía (España)
}

\author{
Standardization of geospatial data of water sources and \\ springs collected in the Andalusian Gazetteer (Spain)
}

\author{
María Teresa Garrido Borrego' \\ Cristina Torrecillas Lozano² \\ Irene García Benabad ${ }^{3}$ \\ Lucia Romero Cardenas ${ }^{4}$ \\ Cora Torrecillas Nicolás ${ }^{5}$
}

Recibido 30 de enero de 2021; aceptado 06 de abril de 2021

\section{RESUMEN}

Los nomenclátores requieren de topónimos oficiales o normalizados por un organismo competente según los estándares, las recomendaciones y las normativas aplicables. El Nomenclátor Geográfico de Andalucía (NGA) es un registro georreferenciado, en continua actualización, conforme a la directiva INSPIRE. Tiene como objetivo ser la base de datos toponímica de referencia de Andalucía, una de las comunidades autónomas de España. El control de calidad de la toponimia es una tarea compleja en la que intervienen problemáticas como la ausencia de los límites físicos de una entidad geográfica (p.e. un paraje natural), la inexistencia de topónimos oficiales o normalizados de la

Instituto de Estadística y Cartografía de Andalucía, España, correo electrónico: mariat.garrido@juntadeandalucia.es. ORCID: https://orcid.org/0000-0003-0617-3773

Departamento de Ingeniería Gráfica, Escuela Técnica Superior de Ingeniería, Universidad de Sevilla, correo electrónico: torrecillas@us.es. ORCID: https://orcid.org/0000-0002-3650-3174

Fundación de Investigación de la Universidad de Sevilla, España, correo electrónico: irenegarciabenabad@hotmail.com

Fundación de Investigación de la Universidad de Sevilla, España, correo electrónico: luciaromerocardenas@hotmail.com

Fundación de Investigación de la Universidad de Sevilla, España, correo electrónico: cora.t.n@gmail.com 
misma, la necesidad de realizar un estudio onomástico individualizado o la desaparición de aquellas personas que conocen el lugar y su historia. Todo ello dificulta la verificación y normalización toponímica. Cada vez es más común que los nomenclátores, como el NGA, se vinculen con otros conjuntos de datos geoespaciales, reutilizando sus nombres geográficos para su enriquecimiento y actualización. A la espera de la aplicación de identificadores únicos, permanentes y universales para las entidades geoespaciales, el nombre y su localización, más su tipología, son los elementos esenciales en la vinculación entre estos conjuntos de datos. Se presentan en este artículo los problemas, soluciones y las propuestas de normalización, siguiendo la norma técnica NTCA 02-021, adoptadas en la vinculación anual del NGA con la base de geodatos "Conoce tus Fuentes", un catálogo online colaborativo de manantiales y fuentes de Andalucía. Este proceso está permitiendo transmitir la importancia de la normalización, ejemplificada en esta tipología de nombres, y como la existencia de identificadores únicos y persistentes contribuye positivamente en las vinculaciones anuales entre dicho proyecto y el nomenclátor andaluz.

Palabras clave: toponimia, normas técnicas, estandarización.

\section{AbStract}

Gazetteers require official or standardized toponyms by a competent organization according to standards, recommendations and applicable regulations. The Geographic Gazetteer of Andalusia (NGA) is a georeferenced registry, in continuous updating and in accordance with INSPIRE Directive. It aim is to be the reference toponymic database of Andalusia, a Spanish autonomous community. The toponymic quality control of is a complex task involving problems such as the absence of physical limits of a geographical entity (e.g. a natural site), the non existence of official or standardized toponyms, the need to carry out an onomastic study or the disappearance of people who know the places and its history. All of this make toponymic verification and its standardization even more difficult. It is increasingly common for gazetteers, such as the NGA, to be linked to other geospatial datasets, reusing their geographic names for enrichment and updating. Without the application of universal identifiers, unique and permanent, for entities, its name and its location, plus its typology, are essential elements in the linkage between these datasets. This article presents some problems, solutions and standardization proposals, following the NTCA 02-021 technical standard, adopted in the annual linking of NGA with the geodatabase "Conoce tus Fuentes", a collaborative online catalogue of water sources and springs of Andalusia. This process is allowing transmitting: the importance of standardization, exemplified in this typology of names; and how the existence of unique and persistent identifiers has helped in the linkages between this project and NGA. 


\section{Introducción}

La toponimia, o conjunto de nombres propios de lugar de un espacio geográfico determinado, es estudiada por la toponomástica y constituye "un fiel reflejo de las realidades del territorio a las que identifica, de las interrelaciones entre los aspectos físicos y humanos del mismo y de sus transformaciones sucesivas" (Tort, 2000). Los topónimos, independientemente de su significación y uso tradicional, son empleados en la elaboración de mapas, censos de población, estudios geográficos, léxico-semánticos, dialectológicos o históricos. Hoy en día, debido a la aplicación de las nuevas tecnologías y, sobre todo, a los mapas en la web, sirven como identificadores geográficos. Así, son usados en la realización de búsquedas de información por geolocalización en Sistemas de Información Geográfica, Sistemas de Información Documentales o en Infraestructuras de Datos Espaciales (López-Pellicer et al., 2006), siendo imprescindibles en el día a día de una gran mayoría de ciudadanos (Arroyo, 2010). Esta búsqueda puede sufrir muchos fracasos si el nombre a buscar no se corresponde con el almacenado en el servicio propio o distribuido de búsqueda (Laborda et al., 2009), se emplee un nombre conocido vulgarmente pero que no es el oficial, la base no es todo lo detallada que se espera o el motor de búsqueda no es lo suficientemente flexible con la coincidencia de caracteres.

Los nomenclátores son grandes bases de datos de topónimos que necesitan cumplir los estándares, las recomendaciones y la normativa autonómica, nacional o europea, en especial la relacionada con INSPIRE (European Union, 2007). Aunque se han planteado procesos para su generación automatizada desde incluso fuentes web no cartográficas (Popescu et al., 2008), la realidad es que requieren de una oficialidad y de la existencia de un organismo que responda por ese nombre.

A nivel nacional, el Instituto Geográfico Nacional está llevando a cabo actuaciones de cara a revisar y armonizar el Nomenclátor Geográfico Básico de España (NGBE) con ayuda de las comunidades autónomas para conformar el Nomenclátor Geográfico Nacional, de carácter oficial y regulado por el Real Decreto 1545/2007 (Vázquez et al., 2015). Asimismo, sin ánimo de exhaustividad, varias comunidades autónomas disponen de publicaciones oficiales, completas o parciales, de nomenclátor, como son Cataluña (Comission de Toponímia de Catalonha, 2006; Rabella, 2004), Navarra (Múgica \& Mendoza, 1993), Aragón, País Vasco, Galicia o Asturias. Valencia (Embuena, 2019) o Andalucía incluyen toponimia oficial y/o normalizada en sus nomenclátores mientras que otras, como Baleares (Fons \& Gomila, 2019; Llauger et al., 2007), están también trabajando en la creación de un nomenclátor geográfico normalizado, armonizado y georreferenciado.

El Instituto de Estadística y Cartografía de Andalucía (IECA) es uno de los principales productores públicos de datos de la Comunidad Autónoma de Andalucía, ubicada en el sur de España. Entre sus productos está el Nomenclátor Geográfico de Andalucía (NGA) que tiene el objetivo de inventariar, normalizar y oficializar progresivamente la toponimia andaluza, así como su difusión. El mismo contiene más de 210.000 entidades con más de 285.000 localizaciones 
de topónimos andaluces clasificados temáticamente. En los últimos 10 años, se ha iniciado el inventario de nombres de entidades albergadas en otras bases de geodatos destinadas a recopilar las denominaciones de una tipología específica (p.e. el patrimonio cultural, el patrimonio natural, los parajes o la hidrografía). Esto permite enriquecer el contenido del NGA, pero ha puesto de manifiesto la existencia de un gran abanico de variantes léxicas de la propia denominación. Aunque en el Sistema Estadístico y Cartográfico de Andalucía (SECA) se dispone de una norma sobre normalización toponímica general, la NTCA 02021, su uso y conocimiento necesita potenciarse y extenderse con más intensidad.

Se presenta en este artículo los problemas, las soluciones y las propuestas de normalización realizadas en el ámbito del NGA en su vinculación periódica con el conjunto de geodatos externo procedente del proyecto "Conoce tus Fuentes" (Castillo \& Sánchez, 2008; Sánchez-Díaz et al., 2012). Este proyecto alberga más de 12.000 denominaciones de entidades geográficas relacionadas con fuentes y manantiales andaluces, junto con más de 1.000 registros referidos a otros puntos relacionados de interés. Para ello, se introduce, en primer lugar, el marco general de las normas técnicas autonómicas de aplicación para, posteriormente, exponer los trabajos de inventario y normalización llevados a cabo en el NGA y, finalmente, ejemplificar la reutilización, vinculación y propuestas de normalización aplicadas en el mencionado conjunto.

\section{Normas Técnicas Cartográficas de Andalucía (NTCA) sobre toponimia}

La labor de normalización es absolutamente necesaria para resolver los problemas de la diversidad o confusión en la denominación de muchos microtopónimos e imprescindible para el buen uso de los nomenclátores. El Decreto 141/2006 de la Junta de Andalucía (Junta de Andalucía, 2007) tenía por objeto la ordenación de la actividad cartográfica de las Administraciones Públicas de Andalucía usando como instrumento fundamental el Plan Cartográfico de Andalucía (PCA). Entre las determinaciones del PCA se encontraban, por una parte, la necesidad de normalización técnica de los procesos de producción y gestión de la Información Geográfica (disposición 23) y, por otra, la calidad como un requisito fundamental para asegurar los mayores niveles posibles de precisión, interoperabilidad y cualidades para ser usada (disposición 24). En 2011 se consolida la integración de los Sistemas Estadístico y Cartográfico de Andalucía en el SECA y se sustituye el PCA por el Plan Estadístico y Cartográfico de Andalucía, aprobándose en agosto de 2013 con una posterior renovación en 2020. Este plan de 2013 incluyó la dotación de un sistema de Normas Técnicas Cartográficas de Andalucía (NTCA) como instrumento para asegurar el rigor técnico, la implantación de procesos de calidad, la transparencia y la simplificación de procesos y productos en las actividades estadísticas y cartográficas, siguiendo las normas y estándares que sean de aplicación en su artículo 23 (IECA, 2011). 


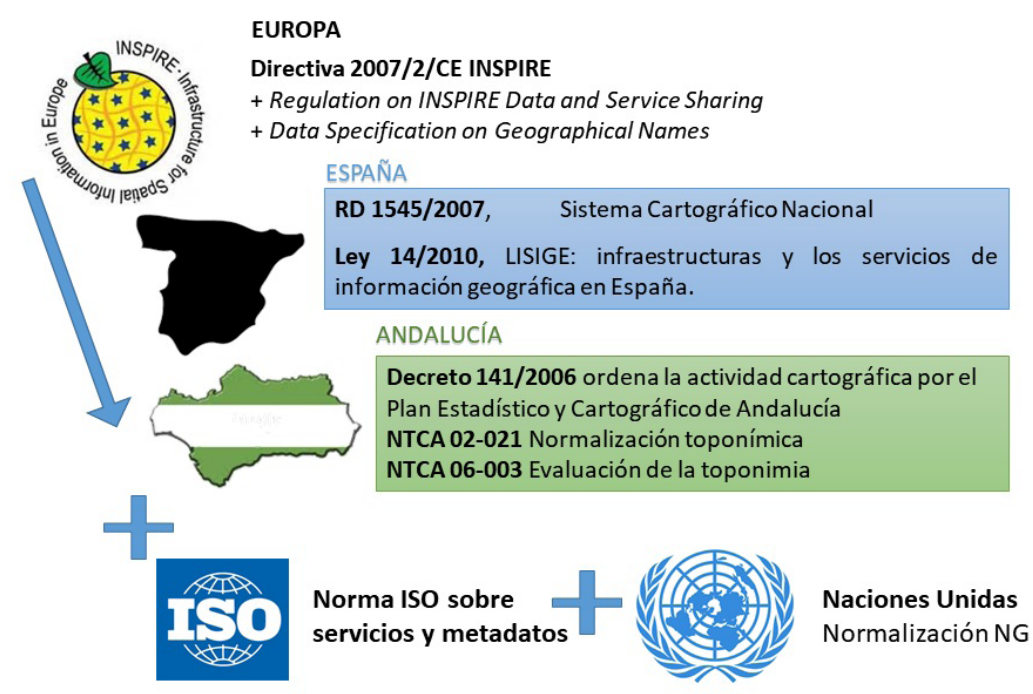

Figura 1. Normativas y recomendaciones toponímicas de aplicación en el NGA. Fuente: elaboración propia.

La normativa y recomendaciones de aplicación en el NGA desde un ámbito global, pasando por el nacional, hasta llegar al autonómico, se muestran en la Figura 1. Hay que destacar las dos normas técnicas autonómicas, aún en fase de borrador, que afectan directamente a la toponimia: la norma NTCA 02-021 sobre la normalización toponímica (Garrido Borrego \& Torrecillas Lozano, 2013b) y la norma NTCA 06-003 sobre la evaluación de la toponimia (Toscano Domínguez, 2013).

\subsection{NTCA 02-021: Normalización toponímica}

La norma NTCA 02-021 sobre normalización toponímica tiene como finalidad establecer las especificaciones técnicas que han de regir en la normalización de la toponimia de Andalucía para proporcionar homogeneidad al tratamiento y uso de los nombres geográficos en la cartografía, infraestructuras de datos espaciales, nomenclátores, productos y servicios geográficos que se realizan dentro del SECA (Garrido \& Torrecillas, 2013a). Sigue las recomendaciones de las Conferencias de las Naciones Unidas para la Normalización de los Nombres Geográficos (Naciones Unidas, 2007), con los siguientes principios de normalización:

a. Principio de univocidad: establecimiento de una forma escrita única de uso preferente para cada nombre geográfico, sin menoscabo de recoger el resto de nombres variantes.

b. Principio de claridad y precisión: referencia inequívoca en las denominaciones de las entidades geográficas para evitar dudas y confusión.

c. Principio de organización rectora y coordinación administrativa: el IECA es el responsable del inventario, normalización y difusión de la toponimia 
de la Comunidad Autónoma en colaboración con el resto de organismos estatales, autonómicos y locales competentes en razón de la materia.

d. Principio de oficialidad: respeto a los nombres oficiales o normalizados por las autoridades competentes, debiéndose consultar los registros y nomenclátores toponímicos de referencia.

e. Principio de procedimiento administrativo: establecimiento de un procedimiento normalizado para la propuesta de nuevos nombres, la modificación y la cancelación de nombres existentes.

f. Principio de prioridad: preferencia de los nombres locales tradicionales y uso público consolidado.

La NTCA 02-021 sigue, además, las siguientes directrices generales:

a. La normalización se refiere a las formas escritas de los nombres, no a la forma oral; para conseguirlo se deben observar unas reglas mínimas convencionales, a fin de dar congruencia y uniformidad a la escritura, así como al uso de los nombres geográficos andaluces, que además deben tratarse de acuerdo a las reglas gramaticales del idioma español.

b. La forma ortográfica de los topónimos se establece a partir de la pronunciación genuina actual y de acuerdo con la documentación más antigua cuando haya vacilaciones, variantes gráficas o dificultad para determinar la forma correcta. En caso de conflicto entre la forma antigua y la actual se ha de respetar la forma evolucionada y actual, conocida y usada por los habitantes del lugar.

c. El tratamiento sistemático de los nombres no debe ser motivo para la supresión de elementos significativos que afecten al término específico del topónimo.

Respecto a las directrices específicas, hay que señalar, en primer lugar, que los topónimos o nombres geográficos están compuestos por un término genérico y un término específico. El término genérico es la parte de un topónimo que identifica de manera general la naturaleza de la entidad denominada, p.e. Fuente de la Reina, Río Guadalquivir, Sierra Nevada, Embalse de Bornos. El término específico es la parte de un topónimo que identifica de manera particular la entidad geográfica denominada, p.e. Sierra Mágina, Río Blanco, El Álamo, Los Altos. Las directrices concretas que afectan a ambos términos son consultables en el texto de la propia norma y hacen referencia al uso de abreviaturas, siglas, acrónimos y símbolos convencionales, acento, aglutinación, artículo, conjunción, exónimo, genérico aislado y término descriptivo, guion, mayúscula y minúscula, nombres de empresas privadas y marcas registradas, números y fechas, paréntesis, preposición “de”, puntuación, término genérico y variantes gráficas.

\subsection{NTCA 06-003: Evaluación de la Toponimia}

La norma NTCA 06-003 sobre la Evaluación de la Toponimia tiene como propósito establecer la metodología detallada para realizar la evaluación de la calidad de la toponimia de los diferentes trabajos sobre información geográfica que se realizan dentro del SECA. La presente norma tiene por objeto específico: 
a. Definir el procedimiento de la evaluación de la toponimia.

b. Detallar los pasos del proceso para realizar la evaluación de la calidad de la toponimia.

c. Desarrollar las medidas de la calidad e indicadores para la evaluación de la calidad de la toponimia.

d. Definir una plantilla para documentar los resultados del proceso de evaluación de la calidad de la toponimia.

e. Desarrollar las medidas para la evaluación de los informantes.

La norma contiene un conjunto de pruebas de conformidad basadas en las normas ISO: UNE-EN ISO 19113 Información geográfica. Principios de calidad; UNE-EN ISO 19114 Información geográfica. Procedimientos de evaluación de la calidad; UNE-EN ISO 19138 Información geográfica. Medidas de calidad de los datos. La NTCA 06-003 es difícil de aplicar al 100\%, como podría ocurrir con otros datos geoespaciales, debido a casuísticas como, por ejemplo:

a. El desconocimiento o la falta de límites tangibles en algunos elementos naturales como los parajes o aquellos ríos que cambian su denominación a lo largo de su recorrido, sin tener claro ese límite.

b. La presencia u omisión de artículos, así como el uso de mayúsculas sin tildes que entorpecen la validación del propio nombre por la duda en la existencia o no de la misma.

c. El uso de diferentes genéricos para un mismo elemento (parroquia por iglesia, cortijo por hacienda).

La aplicación correcta de esta norma requiere disponer de recursos suficientes, no siempre disponibles, para los trabajos de campo correspondientes además de un conjunto de reglas de normalización específicas para validar cada tipo de topónimo.

\section{El Nomenclátor Geográfico de Andalucía}

El proyecto del Nomenclátor Geográfico de Andalucía se inició en 2008 con el objetivo de diseñar y crear un esquema de base de datos de referencia única de los nombres geográficos andaluces, que fuera mantenida, consultada y difundida (Garrido \& Torrecillas, 2011). Es un nomenclátor dinámico, tanto en el inventario de datos de diferentes fuentes como en su progresiva normalización por tipología. Consta de una base de datos que sigue el Modelo de Nomenclátor de España (Rodríguez et al., 2006) y las especificaciones de Inspire (INSPIRE Thematic Working Group Geographical Names, 2009). Contiene, entre otros atributos, la denominación de entidades geográficas, sus diversas localizaciones, su clasificación temática y el estado de normalización toponímica en el que se encuentra la entidad (Oficial, Normalizado, No Normalizado o No Disponible). La aplicación que gestiona el NGA permite administrar, de una forma integral y ágil, el inventario y la normalización de la toponimia andaluza, así como su difusión a través de sus servicios web: el Buscador de Nombres Geográficos (BNG) y los servicios WFS (Garrido \& Torrecillas, 2009, 2014) y WMS (véase apartado "Disponibilidad de datos"). Además, es reutilizado por otras aplicaciones como la Infraestructura de Datos Espaciales de Andalucía, 
más conocida como IDEAndalucia (Sánchez \& Torrecillas, 2003), siendo posible solicitar correcciones por parte de la propia ciudadanía, una parte importante en cualquier nomenclátor (Portolés et al., 2013).

\subsection{Inventario, normalización y coordinación toponímica en el NCA}

EI NGA fue generado inicialmente a partir del vaciado toponímico de la cartografía oficial autonómica a escala 1:10.000 recogida en la Base de Datos de Toponimia 10.000 (BTA10 2004-2006). Este corpus principal se ha ido completando con los topónimos de otras bases de datos, cartográficas o georreferenciadas, producidas por diferentes organismos estatales, autonómicos y locales. La reutilización de dichas fuentes está permitiendo actualizar y completar datos de poblaciones, edificaciones rurales, patrimonio histórico, parajes catastrales, hidrografía, patrimonio natural o infraestructuras. Con estos trabajos, intensificados en los últimos tres años, se dispone del inventario de más de 210.000 entidades con más de 285.000 localizaciones con sus nombres preferentes respectivos, además de algunos nombres variantes de los mismos.

Entre los últimos trabajos de inventario llevados a cabo en el NGA hay que destacar, por su volumen, la incorporación reciente de los parajes de las bases de datos catastrales. Esta incorporación se ha llevado a cabo tras realizarse un estudio que determinó una complementariedad alta entre ambas bases de datos, lo que indicaba que el nivel de coincidencia entre ambas era bajo $(21,7 \%)$ (García et al., 2010). Este proceso de inventario finalizó en 2020 tras analizar 3,5 millones de textos en Andalucía que han producido casi 35.000 altas en el NGA.

Estos topónimos son normalizados de acuerdo con la NTCA 02021 y coordinados, en la medida de lo posible, a través de informes al productor, con los organismos respectivos con objeto de avanzar en la normalización y armonización consensuada de la toponimia andaluza. Así mismo, en función del avance de los trabajos de compleción del inventario, su normalización y los recursos disponibles, se realizará una nueva evaluación de la calidad toponímica del NGA de acuerdo con la NTCA 06-003.

Respecto a los trabajos de coordinación toponímica en materia de normalización, el primer proceso de conjunto llevado a cabo comenzó en 2006, colaborando con la Comisión Especializada de Nombres Geográficos del Consejo Superior Geográfico en la normalización consensuada con las distintas Comunidades Autónomas del Nomenclátor Geográfico Conciso de España (NGCE), que fue actualizado posteriormente en 2011-2012 (Figura 2). A este proceso, le siguió otro gran proceso en curso, iniciado en 2013, de normalización de la toponimia referida a los asentamientos poblacionales (o de otro tipo como la actividad industrial o de determinados servicios o equipamientos) en colaboración con las diputaciones y ayuntamientos andaluces que verifican los topónimos propuestos o proponen otros nuevos. Este trabajo recibe el nombre, a partir de 2016, de Inventario Toponímico de Asentamientos de la Comunidad de Andalucía o Proyecto ITACA (Garrido et al., 2018) y está dando grandes resultados, tanto desde el punto de vista del inventario toponímico como de su verificación y normalización. Se espera obtener una base de referencia ampliamente demandada por parte de la propia administración, para sus tareas 
de planificación y ordenación territorial, como por parte de multitud de usuarios (Gullón et al., 2016).

Desde 2018, se está realizando en colaboración con el Instituto Geográfico Nacional, la armonización del Nomenclátor Geográfico Básico de España (NGBE) con el NGA (Vázquez et al., 2015). Es un trabajo lento dado el detalle de las fuentes de referencia, la cartografía 1:25.000 y 1:10.000, que requiere de recursos suficientes para abordar una gran cantidad de topónimos, algunas veces desactualizados y sustituidos por otros nuevos, capturados erróneamente desde el trabajo de campo (debido, a veces, a errores de transcripción causados por el habla de la zona) o en la digitalización (dónde una "o" en ocasiones es cruzada por una curva de nivel y parece una "a" o una "e", o p.e. una "r" puede parecer una " $n$ ") o simplemente han sido omitidos.

Paralelamente, otras fuentes de datos a nivel autonómico que se están reutilizando en el inventario y normalización toponímica en el NGA son “Conoce tus Fuentes" (Sánchez-Díaz et al., 2012), la base de datos de bienes inmuebles (Muñoz, 2001; Olvera \& Gutiérrez, 2014) o las vías pecuarias delimitadas y publicadas en BOJA por la Consejería competente en Medio Ambiente. Al ser todas estas bases geoespaciales productos vivos, a la resolución de las incidencias entre los conjuntos de datos, se le une un protocolo de actualización, generalmente con un ciclo anual.

No deben olvidarse los trabajos realizados a diario sobre incidencias encontradas entre otros productos cartográficos del IECA, como son la Base Cartográfica de Andalucía (BCA), ${ }^{1}$ los Datos Espaciales de Referencia de Andalucía $(\text { DERA })^{2}$ o el Callejero Digital de Andalucía Unificado (CDAU) ${ }^{3}$ (Figura 2).

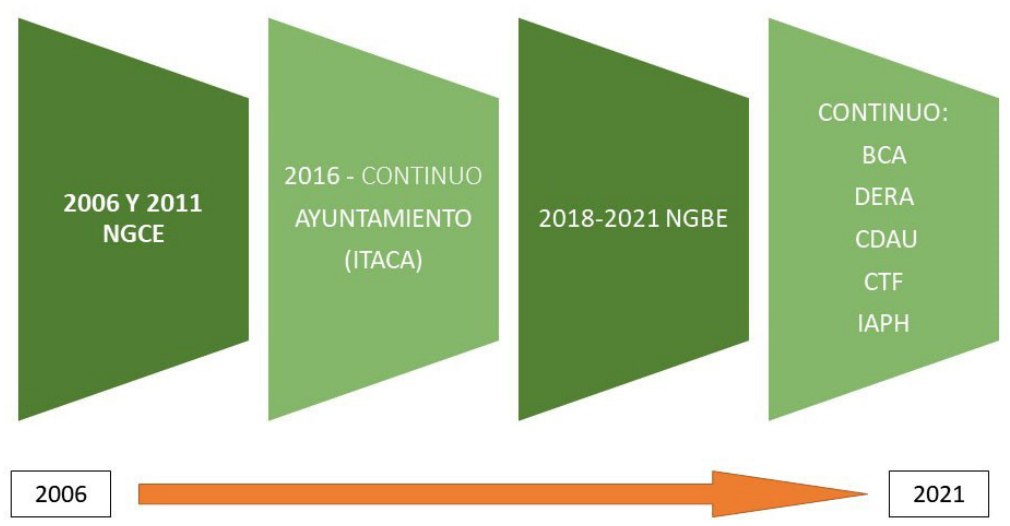

Figura 2. Trabajos de normalización y coordinación toponímica en el periodo 2006-2021. Fuente: elaboración propia. 


\subsection{Problemática en la reutilización de bases de datos georreferenciadas}

El continuo aumento en la creación de bases de datos de elementos geoespaciales por parte de las entidades responsables de los mismos hace que la localización y actualización, espacial o denominativa, de una entidad, por ejemplo de servicios como un museo, un colegio o las dependencias de una institución pública, pase a ser responsabilidad del organismo propietario o gestor de dicha entidad (Caturla, et al., 2014). Esto hace que cada vez sea más común tener que recurrir a diferentes bases de datos para realizar un estudio o trabajo, siendo inevitable la vinculación entre diversos conjuntos de datos referidos a una misma tipología de entidad. Lo que aparentemente podría parecer una tarea sencilla, mediante la unión de campos comunes o mediante una operación espacial de intersección entre las capas geoespaciales, se complica debido fundamentalmente a aspectos como estos:

a. Localización: existe distinta precisión en la localización de los elementos de las distintas bases de datos; generalmente debido a la escala del mapa usada en la localización o a la precisión del instrumental topográfico empleado para dotarlos de coordenadas (GNSS de poca precisión) (Figura 3a).

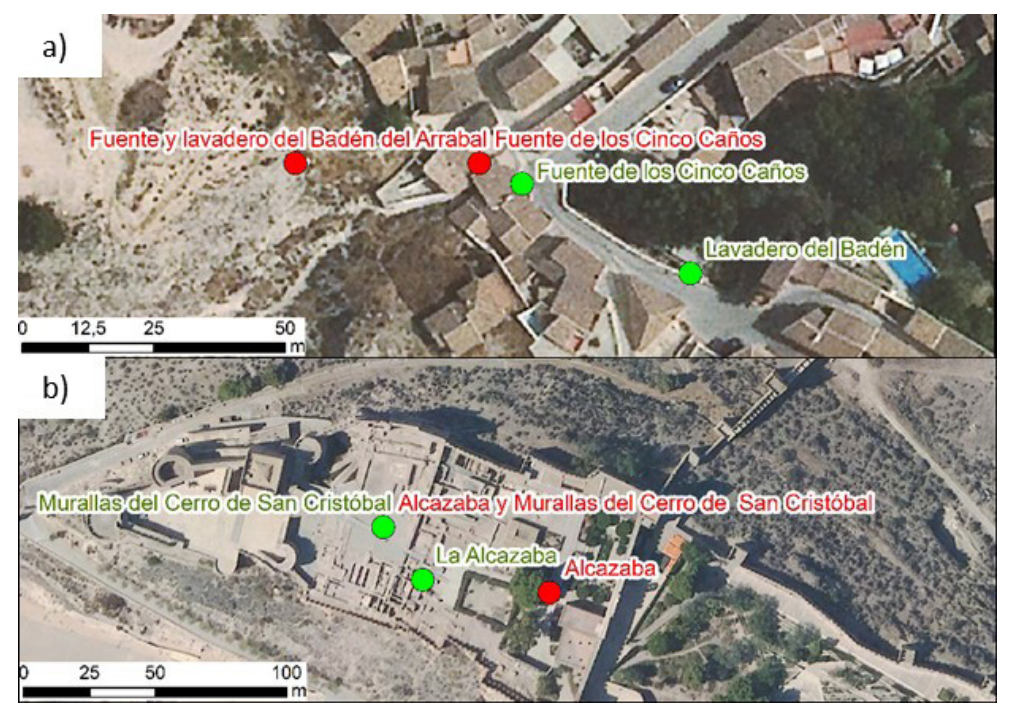

Figura 3. Ejemplos de problemas en el contraste entre bases de geodatos relativas a distinta precisión en la localización y agrupación de más de una entidad geográfica en el nombre. En verde, los datos del NGA y en rojo, otras bases de datos (CTF e IAPH): a) Se muestra una falta de precisión en la localización del Lavadero del Badén y la agrupación de dos entidades (Fuente y lavadero...); b) Se muestra, por un lado, la agrupación de dos entidades (Alcazaba y Muralla...), y por otro, la repetición del elemento Alcazaba.

Fuente: elaboración propia. 
b. Nombre: se emplean diferentes nombres para el mismo elemento, debido a omisiones o diferencias en el término genérico del topónimo (p.e. arroyo por barranco); diferencias en el término específico (más o menos completo, con dos nombres unidos, uso de abreviaturas, totalmente diferentes...); o diferencias en ambos términos, genérico y específico, que lo forman. En la Tabla 1 pueden verse diferentes ejemplos de esta casuística.

Tabla 1. Ejemplo de distintos nombres para una misma entidad hidrográfica entre el Ministerio de Transición Ecológica y el Reto Demográfico (MITECO), en mayúscula y con geometría lineal, y NGA, en minúscula y con geometría puntual

\begin{tabular}{ll}
\hline \multicolumn{1}{c}{ Nombre en MITECO } & \multicolumn{1}{c}{ Nombre en NGA } \\
\hline BARRANCO DEL BARQUERO & Arroyo del Barquero \\
\hline ARROYO DEL SIBUSCO & Regato del Sibusco \\
\hline ARROYO O GARGANTA DE JUAN DE SEVILLA & Arroyo de Juan de Sevilla \\
\hline ARROYO DE MINGO YUSTRE & Arroyo de Mingo \\
\hline BARRANCO DE LAS HILAS O DE TORRE SERENA & Barranco de las Hilas \\
\hline ARROYO DEL MORO GENIL & Barranco del Moco \\
\hline
\end{tabular}

Fuente: elaboración propia.

c. Clasificación y tipo de dato: la clasificación y tipo de dato, mediante un código o un texto, de los elementos es heterogénea y responde a los intereses de cada organismo productor. En ocasiones, hay que igualar elementos de diferentes niveles de clasificación o cambiar, por ejemplo, la tipología del dato de numérico a alfanumérico (algo muy común en los códigos municipales pues suelen ser tratados, unas veces, como cadena de caracteres y, otras, como número).

d. Nivel de detalle de la entidad: se usan diversos niveles de detalle en diferentes bases. Por ejemplo, un castillo puede suponer más de una entidad si se baja al nivel de sus elementos constructivos como podría ser su puerta, una torre o la muralla. Otro ejemplo, podría ser la agrupación de diversos elementos en una sola entidad, p.e. Iglesia y Hospital de La Caridad en una de las bases y en otras, considerada como dos elementos separados: por una parte la iglesia y por el otro el hospital (Figura 3b).

e. Datos cuestionables: hay datos que son válidos en una base de datos pero que no tienen utilidad en otra. Podría ser el nombre de una torre de la que ya no queda ni siquiera ruinas y está totalmente desaparecida. En una cartografía actual no tendría cabida esta entidad, aunque sí en una cartografía histórica o en un nomenclátor como el propio NGA en el que se catalogaría como edificación desaparecida o en ruinas. Otro posible caso sería el de edificaciones de interés arquitectónico que no poseen nombre propio y que se identifican p.e. como Casa Palacio en Calle Ministro Benavides, 
n45. Esta denominación es un descriptivo que no tendría interés para una base de datos de toponimia, pero sí en una que recoja este tipo de edificaciones.

Aparte de los problemas aludidos, la reutilización de datos geoespaciales producidos por distintos organismos es muy beneficiosa como se puede comprobar en dos claros ejemplos: DERA y el NGA, aportando un valor añadido de síntesis o normalización de dichos datos, respectivamente. El DERA es un producto del IECA de referencia, muy empleado y descargado por la recopilación temática que contiene, pero como le ocurre a todos los productos o publicaciones cartográficas, poco referenciado en los estudios o trabajos que hacen uso del mismo (IECA, 2015; López \& Mulero, 2015). Este producto, en general, ofrece una valiosa síntesis de información de distinta procedencia, estructurado en diversas capas de información. El NGA, por su parte, es reutilizado por la IDEAndalucía aportando los nombres geográficos preferentes que deben ser usados en la Comunidad Autónoma.

\subsection{Metodología}

En el caso del NGA, el proceso de incorporación o inventario de toponimia se basa en la reutilización de las bases de datos cartográficas o georreferenciadas producidas por distintas entidades autonómicas, locales o estatales y sigue, simplificadamente, la dinámica mostrada en la Figura 4. Tras la recopilación del nombre (inventario), este es normalizado, coordinado con otros productos geoespaciales, a través de los informes al productor y, finalmente, publicado para facilitar su acceso público por parte de la ciudadanía. Si la base de datos es mantenida y actualizada por la entidad, es necesario, tras ese contraste inicial de incorporación, la definición de un protocolo de actualización que normalmente es de índole anual.

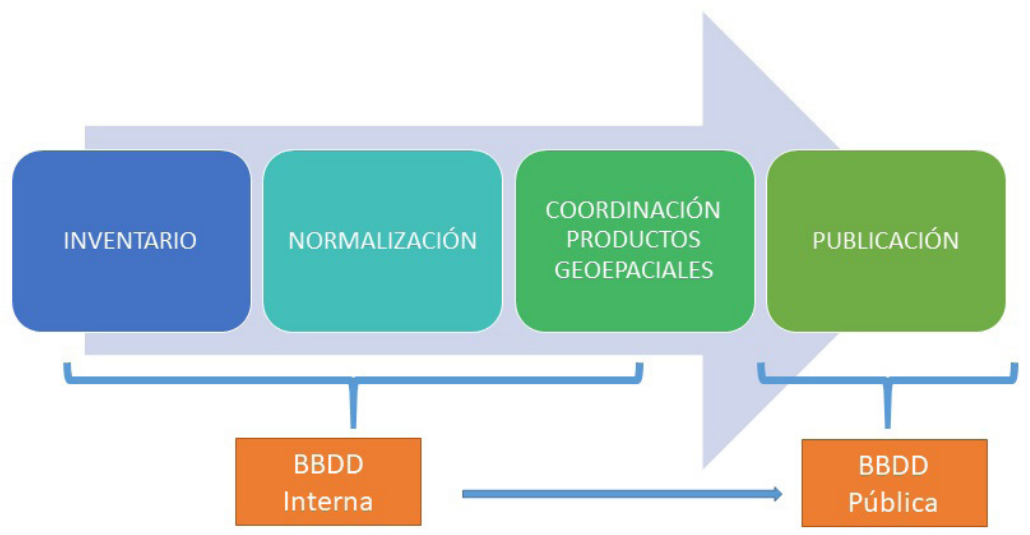

Figura 4. Procedimiento simplificado de la incorporación de topónimos al NGA. Fuente: elaboración propia. 
Es importante para la actualización anual de bases de datos reutilizadas el establecimiento de protocolos de vinculación entre ellas que, generalmente, requieren de varias fases (Figura 5):

a. Tratamiento previo: consistente en el análisis y preparación inicial de la información para poder ser contrastada correctamente.

b. Contraste con NGA: consistente en la comparación de las bases de datos a través de los atributos de nombre+localización+tipo o identificador único permanente, previamente relacionado en el caso de las actualizaciones.

c. Obtención de las coincidencias: consistente en la disposición de nuevas altas y resolución de posibles incidencias de nombre y localización, así como registro del identificador externo, único y permanente, de las entidades procedentes de la base de datos reutilizada, si es que dispone del mismo.

d. Introducción en NGA e informe al productor: consistente en la adaptación al modelo de datos de NGA de las altas y las incidencias resueltas, previamente informadas al productor y contestadas por el mismo.

e. Publicación en los servicios webs del NGA: BNG, WFS y WMS.

El procedimiento automatizado de reutilización y vinculación, por primera vez, entre bases de datos geoespaciales se realiza normalmente usando la suma de tres atributos: nombre+localización+tipología con objeto de acotar los resultadosy los posibles errores debidos a nombres repetidos o no normalizados. Una vez obtenida la vinculación inicial, se realiza una revisión manual consistente en resolver las incidencias de nombre, localización o asignación de tipología.

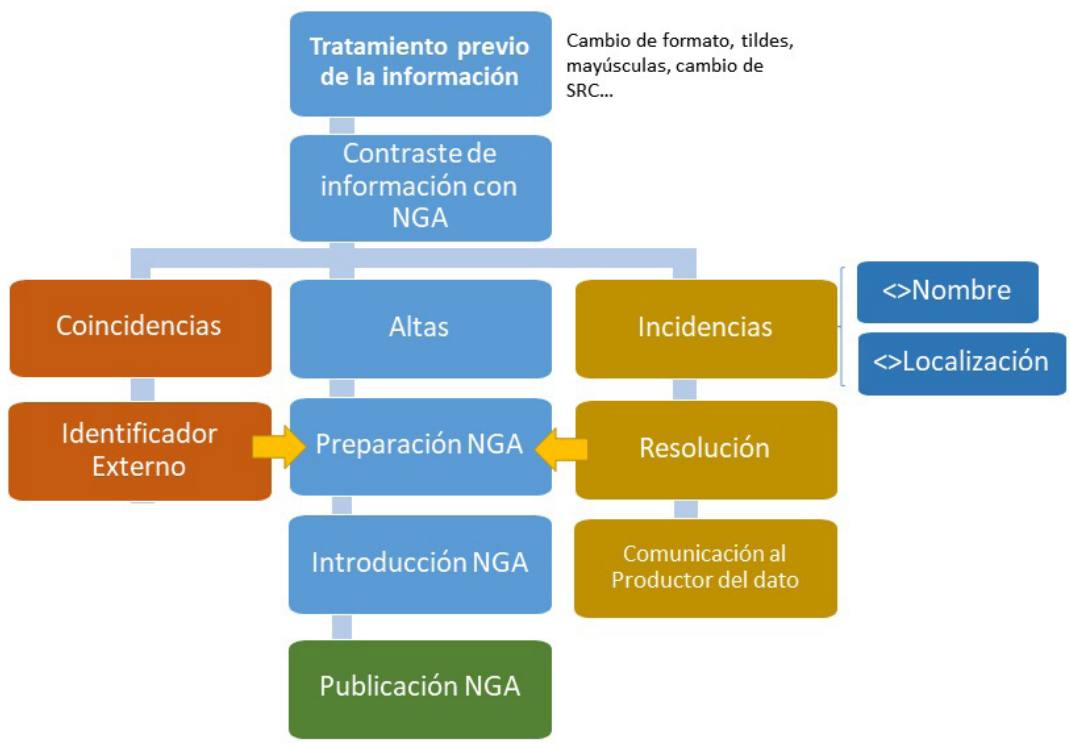

Figura 5. Procedimiento general de incorporación de conjuntos de datos toponímicos. Fuente: elaboración propia. 
A veces, dichas incidencias son motivadas por la falta de persistencia del identificador supuestamente único de la base de datos reutilizada. Establecer una relación estable en el tiempo entre los identificadores únicos del NGA con los identificadores únicos de la base de datos a considerar es un requisito que garantizará una mayor eficacia en las actualizaciones posteriores.

El contraste de nombre se realiza empleando nombres en mayúsculas sin tildes, sin genéricos y con funciones comparativas de texto, como la Levenshtein, ${ }^{4}$ hasta ciertas diferencias de letras u otras métricas (Amón \& Jiménez, 2010), pero siempre se requiere de una revisión manual que resuelva las incidencias detectadas. Asimismo, es necesario vincularlo con una tipología dentro de las más de 400 existentes en NGA (cortijo, iglesia, arroyo...) y aunque la inclusión de genéricos puede ayudar, no siempre es así. Por ejemplo, existen edificios que han cambiado de función pero que siguen manteniendo su nombre anterior por ser el más difundido: tal es el caso del Hospital de los Venerables en Sevilla, que actualmente funciona como centro cultural. Es de destacar que actualmente existen estudios que intentan mejorar estas vinculaciones incluso con Machine Learning, pero sin resultados satisfactorios (Santos et al., 2017).

\section{Experiencia en la reutilización y normalización de datos de fuentes y manantiales}

El proyecto "Conoce tus Fuentes" (CTF) es el primer catálogo de manantiales y fuentes de Andalucía participativo y on line. Comenzó en 2007 de mano de la Agencia Andaluza del Agua de la Consejería de Medio Ambiente y la Universidad de Granada con el objetivo de catalogar y poner en valor las fuentes y manantiales de Andalucía. Estas manifestaciones de agua constituyen un valioso patrimonio ambiental, socio-económico y cultural, que urge conocer y proteger mejor ante nuevas amenazas como el cambio climático y el aumento de explotación de nuestros recursos hídricos (Castillo \& Sánchez, 2008). En agosto de 2012 ya había recibido más de 287.000 visitas y catalogado 6.050 fuentes y manantiales en la comunidad (Sánchez-Díaz et al., 2012). Estas cifras se han incrementado en enero de 2021 a 1.090 .141 visitas y recopilado 12.504 fuentes y manantiales junto con 1.062 registros referidos a otros puntos de interés (OPI).

Este tipo de entidad no suele estar incluido al completo en la cartografía topográfica por su gran detalle pero, indudablemente, es un elemento muy interesante para muchos fines, incluido su valor histórico y patrimonial que ha motivado su incorporación al NGA, aportando casi 13.000 altas de nuevos elementos hídricos omitidos en el MTA10.

\subsection{Cronología y proceso de contraste toponímico}

En 2016 se estudia y plantea la incorporación de los nombres de esta base al NGA, pero es en 2018 cuando comienza una colaboración constante mediante 
peticiones anuales al Instituto del Agua de la Universidad de Granada, que facilita la base de datos con indicaciones de los cambios producidos respecto de la anterior. Por su parte, el IECA comunica, a través del Informe al productor, las dudas y errores detectados, así como información de los criterios de normalización toponímica aplicados, extraídos de la NTCA 02021, para su uso y difusión. En dicho año, la información facilitada se componía de 11.263 registros y 767 lugares de interés. Se detectaron 1.022 incidencias con 686 cambios de nombre, 5 bajas, 34 cambios de localizaciones y 3 registros duplicados en la base de datos de CTF, respecto al análisis anterior. De la actualización de 2018, se envió un informe al productor con dudas sobre 37 fuentes y 5 OPIS (Otros Puntos de Interés) así como se informó de los errores de registros duplicados o existencia de dobles espacios. En 2019, la base facilitada se incrementó en 367 registros y 61 puntos de interés. Lejos de paralizarse la incorporación de elementos a dicho catálogo, este sigue creciendo hasta cifras, en febrero de 2020, de 12.152 fuentes y manantiales y 937 registros de puntos de interés, solicitando aclaración de dudas al productor sobre 24 fuentes y 10 lugares de interés.

El protocolo empleado en la vinculación se describe en la Figura 6 y pasa por preparar los datos de ambas bases de datos para realizar la detección de cambios, es decir, las nuevas altas y las incidencias por modificaciones de nombre o de localización (en ocasiones se mejora la precisión en la localización de una entidad). También es necesario estudiar las bajas o duplicaciones de identificadores de entidades que junto con las modificaciones de nombre/ localización conforman las incidencias que deben ser estudiadas y resueltas con la colaboración del organismo productor.

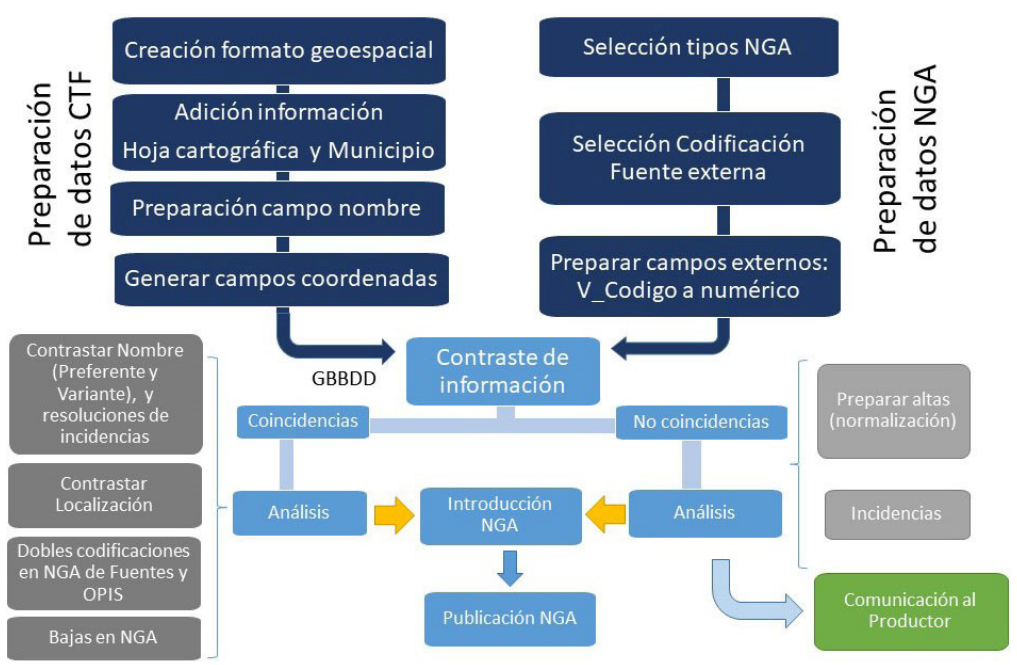

Figura 6. Procedimiento de la incorporación del conjunto de datos "Conoce tus Fuentes" al NGA.

Fuente: elaboración propia. 
En todo el proceso hay que destacar la vinculación de ambas bases de datos, CTF y NGA, a través de sus respectivos identificadores únicos asignados a cada entidad y que facilita enormemente las futuras actualizaciones al reducir la vinculación a través del nombre+localización+tipo mucho más costosa en tiempo y pobre en los resultados. Junto al identificador de CTF, inventariado en NGA en la tabla de codificaciones externas, se añade también, en la tabla de atributos del nombre, el enlace a la web donde puede verse la entidad descrita y fotografiada en el proyecto CTF.

Los principales problemas que se han tenido a lo largo de cuatro contrastes de datos, han sido:

a. Diversidad en la precisión de la localización de sus elementos, teniendo que revisar de forma individualizada y manual la localización de las altas a efectuar.

b. Clasificaciones que deben ser analizadas individualmente para la correcta asignación de tipo de entidad en NGA p.e. "Pozo" unas veces se refiere a un Hidrónimo Puntual, pero otras, sobre todo en la provincia de Jaén, a Patrimonio Minero.

c. Identificadores únicos de CTF duplicados en NGA debido a diferencias de criterio sobre la entidad física. La existencia de un solo registro en CTF se corresponde con varios en NGA, p.e. FUENTE-POZO DE LAS AGUAS en CTF, es entendido como fuente y pozo en NGA, lo que implica duplicidad en los identificadores únicos que debe ser registrada para las sucesivas actualizaciones.

d. Problemas asociados al empleo de mayúsculas en CTF y a la duda de la existencia o no de tildes.

\subsection{Normalización aplicada}

Un resumen de los criterios de normalización toponímica, extraídos de la NTCA 02-021 y del análisis de la base de datos de CTF, forma parte del "Informe al Productor". Este documento es enviado al organismo responsable de la fuente para que lo use de referencia en el alta de nuevos registros o modificación de los preexistentes. La normalización aplicada entre el NGA y CTF sigue las propuestas mostradas en la Tabla 2.

Tabla 2. Propuestas de normalización toponímica para fuentes y manantiales

\begin{tabular}{ll}
\hline \multicolumn{1}{c}{ Propuestas } & \multicolumn{1}{c}{ Ejemplos } \\
\hline Respetar las reglas ortográficas y & Laguna de Ruíz Sánchez > Laguna de Ruiz Sán- \\
gramaticales del español: tildes (incluidas & chez; \\
las mayúsculas), concordancia de género y & Fuente el Collao > Fuente el Collado; \\
número o terminación correcta de las & Fuente del Vinculo > Fuente del Vínculo; \\
palabras. & Fuente del Dornao > Fuente del Dornajo. \\
\end{tabular}




\begin{tabular}{ll}
\hline \multicolumn{1}{c}{ Propuestas } & \multicolumn{1}{c}{ Ejemplos } \\
\hline Utilizar el artículo inicial únicamente en los & Nacimiento > El Nacimiento; \\
topónimos simples, es decir, los formados & El Nacimiento de Laroles > Nacimiento de Laro- \\
por un único nombre común y no en los & les; \\
topónimos compuestos, que ya están & Pilar > El Pilar; \\
suficientemente determinados por el & El Pilar de Santa Ana > Pilar de Santa Ana; \\
término específico. Del mismo modo, se & Pilas > Las Pilas; \\
debe suprimir el artículo inicial en el caso de & Las Pilas de la Fuente del Espino > Pilas de la \\
que el nombre geográfico conste de término & Fuente del Espino; \\
genérico y este corresponda a la naturaleza & Fuente > La Fuente; \\
de la entidad geográfica designada. Todo ello & La Fuente Grande > Fuente Grande; \\
si no contradice el uso tradicional. & \\
\hline
\end{tabular}

Realizar la contracción propia de preposición seguida de artículo.

Laguna de el Chaparral > Laguna del Chaparral; Lavadero de el Chuche > Lavadero del Chuche.

Evitar el uso de topónimos compuestos por más de un nombre unidos por la conjunción disyuntiva "O" o copulativa "y".
Pozo y Molino de los Chorros de Alberto > Pozo de los Chorros de Alberto / Molino de los Chorros de Alberto (dos entidades distintas); Presa y Molino de Sofío > Presa de Sofío / Molino de Sofío (dos entidades distintas)
Evitar los nombres con guion, limitándose a casos muy justificados.
Pozo-Abrevadero de la Medina> Pozo

de la Medina / Abrevadero de la Medina (dos entidades distintas); Fuente de las Cochineras de la PortuguesaZanona > Fuente de las Cochineras de la Portuguesa Zanona.

El Pilar;

La Fuente;

Las Pilas;

El Nacimiento;

La Noria.

Manantial en Barranco del Lobo >

Manantial del Barranco del Lobo;

Manantial en el Arroyo Zapatero >

Manantial del Arroyo Zapatero;

Tornajos del Picón (Sur)> Tornajos del Picón;

Laguna del Camino Palma-Fuentepalmera

> Laguna de Almerique

Pozo Manante del Cortijo Cañada Mahón>

Pozo del Cortijo Cañada Mahón.

\section{tivos}

dad. 


\begin{tabular}{|c|c|}
\hline Propuestas & Ejemplos \\
\hline $\begin{array}{l}\text { Utilizar la numeración I, II, III... } \\
\text { en los casos en los que existe } \\
\text { una Fuente/OPI con el mismo } \\
\text { nombre para evitar confusión y ser } \\
\text { coherente con la numeración. }\end{array}$ & $\begin{array}{l}\text { Fuente de los Aserradores / Fuente de los } \\
\text { Aserradores II > Fuente de los Aserradores } \\
\text { I/ Fuente de los Aserradores II. }\end{array}$ \\
\hline $\begin{array}{l}\text { Evitar repetir el mismo topónimo en el } \\
\text { mismo término municipal pues genera } \\
\text { confusión, atendiendo al principio de claridad } \\
\text { y precisión descrito en la NTCA 02-021. }\end{array}$ & \\
\hline $\begin{array}{l}\text { Evitar el uso de abreviaturas y } \\
\text { respetar el nombre de la entidad si } \\
\text { es oficial o está normalizado. }\end{array}$ & $\begin{array}{l}\text { Rezume de Coto Ríos> Rezume de } \\
\text { Coto-Ríos (El nombre normalizado } \\
\text { del núcleo urbano es Coto-Ríos), }\end{array}$ \\
\hline $\begin{array}{l}\text { Evitar el uso de doble espacio } \\
\text { entre las palabras. }\end{array}$ & Molino de la Cimbarra > Molino de la Cimbarra. \\
\hline
\end{tabular}

Fuente: elaboración propia.

\section{Conclusiones}

La existencia de nomenclátores ricos en contenido normalizado y/o oficializado propicia que sean usados y consultados como elementos de referencia e incluso que formen parte de búsquedas distribuidas en la web. En el caso del NGA, partiendo de la toponimia incluida en la cartografía oficial 1:10.000, se ha ido enriqueciendo con topónimos procedentes de otras fuentes cartográficas o georreferenciadas que han contribuido también a la normalización de los nombres y a la corrección de errores de denominación o de ubicación, revertiendo en la mejora de la calidad de la propia cartografía autonómica.

La reutilización de bases de datos diferentes requiere establecer vínculos que no son sencillos cuando dependen solo del nombre y la localización, por el uso de nombres no normalizados y localizaciones de más o menos precisión dependiendo de la resolución o escala. La vinculación mejora si se dispone de una clasificación de las entidades por tipologías y, sobre todo, de cara a futuras vinculaciones, de la existencia de identificadores únicos y persistentes, asignados a cada entidad registrada. El cuidado en mantener dicho identificador en el tiempo y su control ante bajas y actualizaciones sería el siguiente requisito, pero es algo que aún no está muy extendido entre las instituciones gestoras de los mismos o no se cumple al $100 \%$.

El valor natural, histórico y patrimonial del proyecto "Conoce tus Fuentes" ha motivado la incorporación al NGA de casi 13.000 altas de nuevos elementos hídricos omitidos en el MTA10 pues dado su nivel de detalle no se incluye al completo en una cartografía topográfica. Al ser un proyecto online colaborativo presenta algunas fluctuaciones de nombre o localización que se van resolviendo con la consulta de otros organismos y con la aplicación de criterios de 
normalización toponímica de la NTCA 02-021, comunicados a CTF en el informe al productor. No obstante, la normalización previa llevada a cabo para su introducción en NGA, no implica su consideración como toponimia con estatus normalizado hasta que no sea validada por un organismo competente, como se está realizando con los asentamientos en el proyecto ITACA, cuyos nombres son validados técnicamente y después institucionalmente, en pleno, por los respectivos ayuntamientos.

Por otra parte, la retroalimentación que supone el "Informe al productor", permite avanzar en la armonización y uso coordinado de nombres preferentes de cada entidad geográfica, independientemente de sus posibles nombres variantes, con el objeto de generar seguridad, evitar la ambigüedad y la confusión en el uso de la toponimia andaluza cada vez más empleada como identificador de búsqueda en los sistemas de información geográficas, infraestructuras de datos espaciales o navegadores.

Finalmente, de acuerdo con las Naciones Unidas, las acciones de normalización permiten reducir el costo global y obtener resultados más coherentes y de mayor utilidad para un gran número de organizaciones gubernamentales y no gubernamentales, así como para el público en general. La difusión de esa normalización, en el caso de la toponimia, no es fácil. Las vinculaciones con otras bases ayudan en esta divulgación de la norma, pero hay que seguir trabajando, no sólo en el inventario y normalización, sino también en la difusión de la propia toponimia normalizada contenida en el NGA, fomentando su reutilización y la colaboración con otros productores de toponimia general o temática del Sistema Estadístico y Cartográfico de Andalucía o del Sistema Cartográfico Nacional, con objeto de lograr una gestión pública más eficaz y coordinada de la toponimia de Andalucía.

\section{Agradecimientos}

Este trabajo no hubiera sido posible sin la existencia de proyectos tan interesantes como "Conoce tus Fuentes". Queremos agradecer a los responsables de este proyecto su buen hacer y disposición con el proyecto del NGA. Así mismo, estos resultados han sido factibles gracias al proyecto PRJ201803302 de la Fundación de Investigación de la Universidad de Sevilla financiado por el Instituto de Estadística y Cartografía de Andalucía. Finalmente, agradecer los comentarios de los revisores anónimos que han contribuido a aclarar y mejorar algunos aspectos de este texto.

\section{Disponibilidad de datos}

Los datos empleados en este trabajo son de libre acceso desde: 


\begin{tabular}{|c|c|c|}
\hline Denominación & Dirección Web & Tipo de servicio \\
\hline $\begin{array}{l}\text { NGA-Buscador de } \\
\text { Nombres Geográficos }\end{array}$ & http://www.ideandalucia.es/nomenclator/ & $\begin{array}{c}\text { Consulta y } \\
\text { descarga parcial }\end{array}$ \\
\hline NGA- servicio WFS & http://www.ideandalucia.es/wfs-nga/services? & $\begin{array}{c}\text { Consulta y } \\
\text { descarga parcial }\end{array}$ \\
\hline $\begin{array}{l}\text { NGA- servicio } \\
\text { WFS-INSPIRE }\end{array}$ & $\begin{array}{l}\text { http://www.ideandalucia.es/ } \\
\text { wfs-nga-inspire/services? }\end{array}$ & $\begin{array}{c}\text { Consulta y } \\
\text { descarga parcial }\end{array}$ \\
\hline NGA- WMS-INSPIRE & $\begin{array}{l}\text { http://www.ideandalucia.es/ } \\
\text { wms-nga-inspire/services? }\end{array}$ & Consulta \\
\hline Conoce tus Fuentes & http://www.conocetusfuentes.com/home.ph & Consulta \\
\hline
\end{tabular}

\section{Bibliografía}

Amón, I., \& Jiménez, C. (2010). Funciones de similitud sobre cadenas de texto: una comparación basada en la naturaleza de los datos. International Conference on Information Resources Management.

Arroyo llera, F. (2010). Creciente interés geográfico por la toponimia. Estudios Geográficos, 71(268), 299-309. https://doi.org/10.3989/estgeogr.0600

Castillo Martín, A., \& Sánchez Díaz, L. (2008). El proyecto Conoce tus Fuentes. Acción, 23. Recuperado de http://proyectoaguas.es/download/Divulgacion/2008.d4.pdf

Caturla, C., Toscano, M. T., \& Torrecillas, C. (2014). Estado de implementación de la subtemática "Utility and Governmental services" INSPIRE en Andalucía (sur de España). V Jornadas Ibéricas de Infraestruturas de Dados Espaciais. Recuperado de https://idus.us.es/handle/11441/65476

Comission de Toponímia de Catalonha (2006). Critèris entara toponímia d'encastre municipau. Recuperado de

https://territori.gencat.cat/ca/01_departament/documentacio/territori-iurbanisme/cartografia/criteris_per_a_la_toponimia_dambit_municipal_criteris/

Embuena Puerta, C. (2019). Nomenclátor toponímico valenciano. Mapping, 194, 6-11. Recuperado de https://dialnet.unirioja.es/servlet/articulo?codigo=6925185

European Union (2007). Directiva 2007/2/CE del Parlamento Europeo y del Consejo de 14 de marzo de 2007 por la que se establece una infraestructura de información espacial en la Comunidad Europea (Inspire). Diario Oficial de la Unión Europea, (L. 108), 1-14. Recuperado de

https://eur-lex.europa.eu/legal-content/ES/TXT/PDF/?uri=CELEX:32013D1082\&from=EN

Fons, M. E., \& Gomila, X. (2019). Sobre la situación de la toponimia oficial en las Illes Balears: el Nomenclátor de Toponimia de Menorca y el futuro Nomenclátor Geográfico de las Illes Balears. Mapping, 28, 48-56.

Recuperado de http://ojs.revistamapping.com/index.php?journal= MAPPING\&page=article\&op=view\&path\%5B\%5D=184\&path\%5B\%5D=54

García Balboa, J. L., Ureña Cámara, M. A., Ariza López, J. F., Garrido Borrego, M. T., \& Torrecillas Lozano, C. (2010). Análisis comparativo entre la base de datos de toponimia 1:10.000 (BTA10) y la toponimia contenida en la base de datos catastral. 1er Congreso Internacional de Catastro Unificado y Multipropósito. 
Jaén. Recuperado de http://coello.ujaen.es/congresos/cicum/ponencias/ Cicum2010.2.14_GarciaBalboa_Analisis_comparativo.pdf

Garrido Borrego, M. T., Nieto Calmaestra, J. A., \& Torrecillas Lozano, C. (2018). Normalización de la toponimia de los asentamientos de población y actividad en Andalucía: El proyecto ITACA. Boletín de la Real Sociedad Geográfica, CLII, 127-144. Recuperado de https://boletinrsg.com/index.php/boletinrsg/article/view/33

Garrido Borrego, M. T., \& Torrecillas Lozano, C. (2009). Interoperabilidad del servicio de nomenclator y catálogo online del Instituto de Cartografía de Andalucía. I/I Jornadas de SIG libre. Universitat de Girona. Servei de Sistemes d'Informació Geogràfica i Teledetecció (SIGTE). Recuperado de https://idus.us.es/handle/11441/65474

Garrido Borrego, M. T., \& Torrecillas Lozano, C. (2011). Nomenclátor Geográfico de Andalucía. Revista PH, 19(77), 92. https://doi.org/10.33349/2011.77.3109

Garrido Borrego, M. T., \& Torrecillas Lozano, C. (2013a). Normalización de Toponimia en Andalucía: la Norma Técnica Cartográfica de Andalucía NTCA 02-021. Mapping, (162), 24-35. Recuperado de https://dialnet.unirioja.es/servlet/articulo?codigo $=4558632$

Garrido Borrego, M. T., \& Torrecillas Lozano, C. (2013b). NTCA_02021 Normalización toponímica. Recuperado de

https://www.juntadeandalucia.es/institutodeestadisticaycartografia/ieagen/ sea/ntca/02_procesos/NTCA_02021_Procesos_Normalizacion_Toponimica_ BORRADOR.pdf

Garrido Borrego, M. T., \& Torrecillas Lozano, C. (2014). Servicios web de toponimia del Instituto de Estadística y Cartografía de Andalucía. Els noms en la vida quotidiana. Actes del XXIV Congrés Internacional d'ICOS sobre Ciències Onomàstiques. https://doi.org/10.2436/15.8040.01.234

Gullón Muñoz-Repiso, T., Merino Martín, J., \& Camón Soteres, L. (2016). Generación de Información Geográfica de Referencia (IGR) de Poblaciones: automatización de procesos. Mapping, (176), 46-57. Recuperado de https://dialnet.unirioja.es/servlet/articulo?codigo=5444591

IECA, Junta de Andalucía (2011). Estado de desarrollo del programa de normas técnicas cartográficas de Andalucía. Recuperado 6 de enero de 2021, de https://www.juntadeandalucia.es/institutodeestadisticaycartografia/ieagen/ sea/ntca/estado.htm

IECA, Junta de Andalucía (2015). Datos Espaciales de Referencia de Andalucía. Recuperado 19 de mayo de 2018, de https://www.juntadeandalucia.es/institutodeestadisticaycartografia/DERA/

INSPIRE Thematic Working Group Geographical Names (2009). D2.8.I.3 INSPIRE Data Specification on Geographical names. Recuperado 31 de octubre de 2019, de https://inspire.ec.europa.eu/id/document/tg/gn

Junta de Andalucía (2007). Decreto 141/2006, de 18 de julio, por el que se ordena la actividad cartográfica en la Comunidad Autónoma de Andalucía. BOJA num. 154, de 9 de agosto de 2006. En Boletín Oficial de la Junta de Andalucía (pp. 8-14). Sevilla. Recuperado de https://www.juntadeandalucia.es/boja/2006/154/1

Laborda, C., Recio, R., Agudo, J. M., \& Rodríguez, A. F. (2009). Una aproximación a la búsqueda distribuida de topónimos. VI Jornadas de la Infraestructura de Datos Espaciales de España (IIDEE), Murcia. 
Llauger Rosselló, J. A., Ordinas Garau, A., \& Planisi Gili, H. (2007). Nomenclàtor de la toponímia major de les Illes Balears. COFUC (Consorci per al Foment de la Llengua Catalana i la projecció exterior de la cultura de les Illes Balears). Recuperado de https://dialnet.unirioja.es/servlet/libro?codigo=766639

López-Pellicer, F. J., Béjar, R., Zarazaga-Soria, F. J., \& Muro-Medrano, P. R. (2006). Aspectos de modelos e infraestructura de servicios para el soporte de un servicio nacional estándar de nomenclátor en web. III Jornadas de Infraestructuras de Datos Espaciales (IIDEE). Castellón de la Plana. Recuperado de http://docplayer.es/96168511-Aspectos-de-modelos-e-infraestructura-de-serviciospara-el-soporte-de-un-servicio-nacional-estandar-de-nomenclator-en-web.html

López, D., \& Mulero, A. (2015). La contribución de los Datos Espaciales de Referencia de Andalucía (DERA) a la investigación geográfica: el caso de las parcelaciones ilegales del municipio de Córdoba. XXIV Congreso de la AGE: Análisis espacial y representación geográfica: innovación y aplicación (195-204). Recuperado de https://dialnet.unirioja.es/servlet/articulo?codigo=7386267

Múgica, M., \& Mendoza, J. L. (1993). De toponimia navarra. Sobre el "Nomenclátor euskériko de Navarra" Aspectos filológicos y sociológicos. Asju, 27(2), 551-574. Recuperado de http://www.ehu.eus/ojs/index.php/ASJU/article/view/8363

Muñoz Cruz, V. (2001). Hacia un Sistema Integrado de Patrimonio Histórico de Andalucía: la base de datos del Patrimonio Inmueble. Revista PH, 37(37), 223. https://doi.org/10.33349/2001.37.1294

Naciones Unidas. (2007). Novena Conferencia de las Naciones Unidas sobre la Normalización de los Nombres Geográficos Nueva York, 21 a 30 de agosto de 2007.

Olvera Lobo, M. D., \& Gutiérrez Artacho, J. (2014). Visibilidad y presencia de los bienes inmuebles de Andalucía en la Web 2.0, 1-11. Recuperado de http://digibug.ugr.es/handle/10481/35872

Popescu, A., Grefenstette, G., \& Moëllic, P. A. (2008). Gazetiki: Automatic creation of a geographical gazetteer. Proceedings of the ACM International Conference on Digital Libraries (pp. 85-93).

Portolés Rodríguez, D., Martínez Cebolla, R., López Martín, F. G., \& Monteagudo Latorre, S. (2013). Incorporación de la participación ciudadana en el aseguramiento de la calidad de un nomenclátor de topónimos. Scire, 19(2), 83-90. Recuperado de https://ibersid.eu/ojs/index.php/scire/article/view/4089/3732

Rabella i Ribas, J. A. (2004). El nomenclàtor a Catalunya. Llengua nacional: publicació de I'Associació Llengua Nacional, 46, 20. Recuperado de https://dialnet.unirioja.es/servlet/articulo?codigo=3382379

Rodríguez Pascual, A., López Rodríguez, E., Abad Power, P., \& Sánchez Maganto, A. (2006). Modelo de Nomenclátor de España v1.2. Madrid.

Sánchez-Díaz, L., Robles-Arenas, V. M., Castillo, A., \& Fernández-Palacios, J. M. (2012). El proyecto Conoce tus Fuentes: cuatro años dando a conocer los manantiales y fuentes de Andalucía. SIAGA (839-848). Cádiz. Recuperado de http://proyectoaguas.es/download/Articulos/2012.a8.pdf

Sánchez Díaz, F. J., \& Torrecillas Lozano, C. (2003). Las infraestructuras de datos espaciales. Mapping, (86), 41-43. Recuperado de http://dialnet.unirioja.es/servlet/extart?codigo $=499659$

Santos, R., Murrieta-Flores, P., \& Martins, B. (2017). Learning to combine multiple string similarity metrics for effective toponym matching. International Journal of Digital Earth, 0(0), 1-26. https://doi.org/10.1080/17538947.2017.1371253 
Tort i Donada, J. (2000). Toponimia y territorio: Los nombres de los núcleos de población de la comarca del Baix Camp, Tarragona, desde una perspectiva onomasiológica. Scripta Nova. Revista Electrónica de Geografía y Ciencias Sociales, 4(4), 67. https://revistes.ub.edu/index.php/ScriptaNova/article/view/196

Toscano Domínguez, M. T. (2013). NTCA_06003 Evaluación de la Toponimia. Recuperado de https://www.juntadeandalucia.es/institutodeestadisticaycartografia/ieagen/ sea/ntca/06_calidad/NTCA_06-003_Evaluaci\%C3\%B3n\%20de\%20la\%20 Toponimia_BORRADOR.pdf

Vázquez Hoehne, A., Rodríguez de Castro, A., Pavo López, M., Montilla Lillo, M., \& Castaño Suárez, A. (2015). Valoración del cotejo del Nomenclátor Geográfico Básico de España con los nomenclátores de las distintas comunidades autónomas. En La Toponimia en el mundo actual. Entre el patrimonio cultural y el dato georreferenciado. IV Jornada de la Comisión Especializada de Nombres Geográficos (CENG). Valladolid: E.T.S.I. en Topografía, Geodesia y Cartografía (UPM). Recuperado de

https://www.mitma.gob.es/recursos_mfom/pdf/D6051C50-B465-4141-B6BB2846918FCE51/131710/10_Valoracion.pdf 